\title{
Differential modulation of innate immunity in vitro by probiotic strains of Lactobacillus gasseri
}

Diomira Luongo ${ }^{1}$, Junki Miyamoto ${ }^{2}$, Paolo Bergamo ${ }^{1}$, Filomena Nazzaro ${ }^{1}$, Federico Baruzzi ${ }^{3}$, Toshihiro Sashihara ${ }^{4}$, Soichi Tanabe ${ }^{2}$ and Mauro Rossi $i^{*}$

\begin{abstract}
Background: Probiotics species appear to differentially regulate the intestinal immune response. Moreover, we have shown that different immune-modulatory abilities can be found among probiotic strains belonging to the same species. In this study, we further addressed this issue while studying L. gasseri, a species that induces relevant immune activities in human patients.

Results: We determined the ability of two strains of L. gasseri, OLL2809 and L13-la, to alter cell surface antigen expression, cytokine production and nuclear erythroid 2-related factor 2 (Nrf2)-mediated cytoprotection in murine bone marrow-derived dendritic cells (DCs) and MODE-K cells, which represent an enterocyte model. Differential effects of L. gasseri strains were observed on the expression of surface markers in mature DCs; nevertheless, both strains dramatically induced production of IL-12, TNF-a and IL-10. Distinctive responses to OLL2809 and L13-la were also shown in MODE-K cells by analyzing the expression of MHC II molecules and the secretion of IL-6; however, both L. gasseri strains raised intracellular glutathione. Treatment of immature DCs with culture medium from MODE-K monolayers improved cytoprotection and modified the process of DC maturation by down-regulating the expression of co-stimulatory markers and by altering the cytokine profile. Notably, bacteria-conditioned MODE-K cell medium suppressed the expression of the examined cytokines, whereas cytoprotective defenses were significantly enhanced only in DCs exposed to OLL2809-conditioned medium. These effects were essentially mediated by secreted bacterial metabolites.

Conclusions: We have demonstrated that L. gasseri strains possess distinctive abilities to modulate in vitro DCs and enterocytes. In particular, our results highlight the potential of metabolites secreted by L. gasseri to influence enterocyte-DC crosstalk. Regulation of cellular mechanisms of innate immunity by selected probiotic strains may contribute to the beneficial effects of these bacteria in gut homeostasis.
\end{abstract}

Keywords: L. gasseri OLL2809, L. gasseri L13-la, Mouse dendritic cells, MODE-K cells, Immunomodulation

\section{Background}

The intestinal microbiota interacts with the local immune system to promote mechanisms of intestinal homeostasis and health. Many studies have provided evidence that probiotics can also effectively modulate the gut immune system in health and disease [1]. In particular, probiotic bacteria influence both the development and regulation of intestinal immune responses and non-immune defenses [2]. The symbiosis between human hosts and gut microbes has risks and benefits for the host organism as bacteria

\footnotetext{
* Correspondence: mrossi@isa.cnr.it

${ }^{1}$ Institute of Food Sciences, NRC, Avellino, Italy

Full list of author information is available at the end of the article
}

continuously challenge intestinal immune homeostasis with microbial-associated molecular patterns (MAMPs). However, the risks of an exaggerated inflammatory response and chronic inflammation are limited by the polarized expression of pattern recognition receptors intracellularly or on the basolateral membrane of epithelial cells (ECs) and dendritic cells (DCs) that intercalate between ECs for direct bacterial uptake [3].

Paradoxically, little information is available regarding probiotics that possess physiologically relevant antioxidant properties. Nevertheless, a large body of evidence confirms that high-grade oxidative stress is one of the crucial players in the pathogenesis of disorders such as inflammatory diseases. Accumulating data suggest that the 
nuclear erythroid 2 p45-related factor 2 (Nrf2) is a key regulatory transcription factor that induces defense-related genes that protect against the deleterious effects of reactive oxygen species (ROS) and that targeted activation of this transcription factor could represent a therapeutic approach for the treatment of inflammatory diseases [4]. Nrf2 is a redox-sensitive, basic leucine zipper transcription factor. During oxidative stress, following detachment from a cytosolic inhibitor (Keap1), Nrf2 translocates to the nucleus and leads to transcription of antioxidant and detoxifying enzymes involved in detoxification and chemoprevention such as glutathione S-transferases (GSTs), NAD(P)H:quinone oxidoreductase (NQO1) and gamma-glutamylcysteine ligase $(\gamma \mathrm{GCL})$, which is the rate-limiting enzyme for glutathione (GSH) synthesis [5]. Bacteria-induced ROS generation greatly influences eukaryotic signaling pathways including those inducing Nrf2 [6,7], and improved Nrf2-mediated protection is associated with beneficial effects elicited by probiotic intake $[8,9]$.

When studying host responses, there is a tendency to focus on individual cell types that comprise the biological barriers to microorganisms to obtain information on a particular cellular reaction to a microbe. Specifically, in vitro studies have focused on interactions between probiotics and enterocytes. The immunomodulatory role of the intestinal epithelium is attracting considerable attention, in addition to its well-known role in barrier function. In analyses of enterocytes, it was shown that Bifidobacterium infantis and Lactobacillus salivarius did not induce proinflammatory responses in human intestinal epithelial cells (IECs) compared with the responses generated by Salmonella typhimurium, suggesting that IECs display immunological unresponsiveness when exposed to LAB [10]. Using a co-culture model including Caco-2 (IEC) and PBMC cells, Haller et al. also observed differential IEC activations between Escherichia coli and LAB strains [11]. Furthermore, Rimoldi et al. reported that the release of pro-inflammatory mediators by IECs in response to bacteria is dependent on bacterial invasiveness and the presence of flagella in a human co-culture system [12]. Other relevant studies have focused on dendritic cells (DCs), canonical antigen-presenting cells, that can effectively induce primary immune responses against microbial infections and other stimuli $[13,14]$. A recent report demonstrated that individual strains from the Lactobacillus group can differentially regulate the expression of surface markers and cytokine production by DCs [15]. By using human DCs as a model, it was shown that bacterial strains belonging to different species display distinct immunomodulatory effects [16]. Moreover, different strains of the same species can also differentially polarize the immune response $[17,18]$. Recently, we have examined this aspect by focusing on $L$. paracasei that we have found to induce the highest maturation degree of DCs among the tested species [19]. In particular, we observed a differential ability of five genetically characterized $L$. paracasei strains to modulate DCs [20]. In this study, we addressed the same question by studying $L$. gasseri. We focused on L. gasseri because this species induces relevant immune activities in human patients [21]. To simulate the interactions occurring in the intestinal mucosa, we challenged in vitro MODE-K cells, a mouse model of enterocyte, and murine bone marrow DCs with two L. gasseri strains. Our work indicated the existence of strain-specific effects of $L$. gasseri. This modulatory activity was found to be associated with the production of bacterial metabolites distinctively impacting both the immune and anti-oxidant properties of IECs and DCs.

\section{Methods}

\section{Bacterial strains and culture conditions}

Lactobacillus gasseri OLLL2809 (from human intestine; deposited in the Patent Microorganisms Depositary, National Institute of Technology and Evaluation, Japan, Accession n. NITE BP-72) and L13-Ia (from raw bovine milk, deposited in the Microbial Culture Collection, Institute of Sciences of Food Production, Italy, Accession n. 13541) were studied. Strain OLL2809 is considered to be a probiotic strain [22], while potential probiotic features of strain L13-Ia, able to resist to simulated gastric and pancreatic digestion, as well as to bovine and porcine bile salts were previously demonstrated [23]. Working cultures were grown in deMan Rogosa Sharpe (MRS) broth (Difco, Detroit, Michigan, USA) for $24 \mathrm{~h}$ at $37^{\circ} \mathrm{C}$ under aerobic conditions without shaking, and these cultures were subcultured twice before use in experiments. The cell concentration of individual strains was evaluated by measuring the optical density at $600 \mathrm{~nm}$ and converting this value to the corresponding $\mathrm{CFU} \mathrm{ml}{ }^{-1}$ value. Before eukaryotic cell challenge, bacterial strains were irradiated with 2800 Gy (Gray) $\gamma$-irradiation (MDS Nordion $\gamma$-cell 1000) to prevent their proliferation.

\section{Antimicrobial activity}

The antimicrobial activity was assessed by using the inhibition halo test. The pathogenic Bacillus cereus (DSM 4313 and DSM 4384), Escherichia coli (DSM 8579) and Pseudomonas aeruginosa species were used as tester strains. The two strains of Lactobacillus gasseri were grown in MRS broth at $37^{\circ} \mathrm{C}$ to $1 \times 10^{6} \mathrm{CFU} \mathrm{ml}^{-1}$. Cells were centrifuged at $5000 \times \mathrm{g}$ for $15 \mathrm{~min}$ at $4^{\circ} \mathrm{C}$ and collected supernatant was filtered through a $0.22 \mu \mathrm{m}$ filter before use for the test. Different volumes of supernatants were spotted onto sterile filter disks with a diameter of $5 \mathrm{~mm}$ that were plated onto TY (Tryptone Yeast extract, Difco) agar plates previously inoculated with the pathogen tester strains. The TY agar plates were then incubated at $37^{\circ} \mathrm{C}$ for $24-48$ hours. DMSO was used as negative control; gentamycin $(8 \mu \mathrm{g} /$ 
disc) and tetracycline $(7 \mu \mathrm{g} /$ disc $)$ were used as positive controls. The test was performed in triplicate.

\section{IEC cell line}

MODE-K cells (H-2 k), a murine small intestinal epithelial cell line [24], were kindly provided by Dr. D Kaiserlian (INSERM, Paris, France). These cells were maintained as adherent cells at $37^{\circ} \mathrm{C}$ in a humidified atmosphere of $5 \%$ $\mathrm{CO}_{2}$ in air in RPMI medium (Sigma, St. Louis, MO) containing $25 \mathrm{mM}$ HEPES, $1 \%$ nonessential amino acids, $0.055 \%$ sodium pyruvate, $10 \% \mathrm{FCS}$, and $4 \mathrm{mM}$ L-glutamine (complete RPMI medium). Cells were detached before analysis using a solution of $0.25 \%$ trypsin in $0.5 \mathrm{mM}$ EDTA (Sigma, St. Louis, MO). In some experiments, MODE-K cells were treated with recombinant murine TNF- $\alpha\left(5 \mu \mathrm{g} \mathrm{l}^{-1}\right.$, PharMingen, San Diego, CA) for $24 \mathrm{~h}$.

\section{Mice}

B10.M mice were maintained under pathogen-free conditions at the animal facility of the Institute of Food Sciences. Mice were used at the age of 6-12 weeks and were euthanized by inhalation of anesthesia with isoflurane. These studies were approved by the National Institutional Review Committee.

\section{Isolation of bone marrow-derived dendritic cells}

Murine DCs were generated according to a previously published method [25]. In brief, bone marrow cells from the femurs and tibiae of mice were flushed and bone marrow cell aliquots $\left(2 \times 10^{6}\right)$ were diluted in $10 \mathrm{ml}$ of RPMI 1640 medium supplemented with $25 \mathrm{mM}$ HEPES, antibiotics (penicillin $100 \mathrm{IU} \mathrm{ml}^{-1}$; streptomycin $100 \mathrm{IU} \mathrm{ml}^{-1}$ ), 10\% fetal calf serum and $20 \mathrm{ng} \mathrm{ml}^{-1}$ granulocyte-macrophage colonystimulating factor (GM-CSF) (culture medium) before being seeded in 100-mm petri dishes (Falcon, Heidelberg, Germany). On day 3, $10 \mathrm{ml}$ of culture medium was added, and on day 7, $10 \mathrm{ml}$ of the culture medium was replaced with freshly prepared medium. On day 9, non-adherent DCs were harvested by gentle pipetting. Cell aliquots $(1 \times$ $10^{6} \mathrm{ml}^{-1}$ ) were then placed in 24-well plates and incubated in culture medium with $5 \mathrm{ng} \mathrm{ml}^{-1}$ GM-CSF in the presence of $1 \mu \mathrm{g} \mathrm{ml}^{-1}$ LPS for $6 \mathrm{~h}$ (LPS pulse) to induce the maturation of iDCs. Cell viability was microscopically evaluated by dye-exclusion test using Nigrosin ( $1 \%$ solution) and found $\geq 90 \%$ live cells in all experiments.

\section{Microbial challenge}

Confluent epithelial MODE-K cell monolayers or DCs $(1 \times$ $10^{6} \mathrm{ml}^{-1}$ ) were incubated for $24 \mathrm{~h}$ with irradiated bacteria resuspended in complete RPMI medium at a 30:1 bacteria: eukaryotic cell ratio. Following incubation, cells were analyzed by Nigrosin and $\geq 90 \%$ live cells were still found. Conditioned media were centrifuged at $10000 \times \mathrm{g} 10 \mathrm{~min}$ to eliminate any residual cells and cell debris and supernatants stored at $-80^{\circ} \mathrm{C}$. No $\mathrm{pH}$ change occurred in the medium after $24 \mathrm{~h}$ of bacteria incubation. In crosstalk experiments, iDCs were treated with supernatants from the MODE-K cell culture for $24 \mathrm{~h}$, then LPS-pulsed and cultured for additional $24 \mathrm{~h}$ in complete RPMI medium.

\section{FACS analysis}

DCs were stained with phycoerythrin (PE)- or fluorescein isothiocyanate (FITC)-conjugated Abs (BioLegend, San Diego, CA, USA) against CD11b, CD11c, CD40 and CD80. MODE-K cells were analyzed for MHC class II expression using a FITC-conjugated goat anti-mouse antibody (BioLegend). Cell staining was analyzed using a CyFlow Space flow cytometer (Partec, Munster, Germany) and Flowjo software (Tree Star Inc., Ashland, OR, USA). For each Ab, an isotype control of the appropriate subclass was used.

\section{Analysis of cytokine production}

Supernatants from DCs cultures were analyzed for IL12 , TNF- $\alpha$ and IL-10 protein levels, whereas MODE-K cell supernatants were analyzed for IL-6 expression by sandwich-type ELISA. First, $100 \mu \mathrm{l}$ of capture antibody solution (BioLegend) were dispensed into each well of a 96-well plate (Nunc Maxisorb; eBioscience Inc., San Diego, CA) and incubated overnight at $4^{\circ} \mathrm{C}$. After the removal of the capture antibody solution, $100 \mu \mathrm{l}$ of PBS supplemented with $2 \%$ BSA (blocking buffer) were added to each well and incubated at room temperature for $2 \mathrm{~h}$. Next, cytokine standards and samples diluted in blocking buffer supplemented with $0.05 \%$ Tween-20 were added to the respective wells and incubated overnight at $4^{\circ} \mathrm{C}$. At the end of the incubation, after three washings steps with PBS supplemented with $0.05 \%$ Tween-20, $100 \mu \mathrm{l}$ of biotinylated antibody solution were added to the wells and incubated for $2 \mathrm{~h}$ at room temperature. After three washing steps, streptavidin-horseradish peroxidase conjugate (1:2000 dilution; Biolegend) were then added to the wells and incubated for $1 \mathrm{~h}$ at room temperature. Finally, after washing, $100 \mu \mathrm{l}$ of $63 \mathrm{mM}$ $\mathrm{Na}_{2} \mathrm{HPO}_{4}, 29 \mathrm{mM}$ citric acid ( $\mathrm{pH}$ 6.0) containing $0.66 \mathrm{mg} \mathrm{ml}^{-1}$ o-phenylenediamine/ $\mathrm{HCl}$ and $0.05 \%$ hydrogen peroxide were dispensed into each well, and the wells were allowed to develop. The absorbance was read at $415 \mathrm{~nm}$ and the cytokine concentrations were calculated using standard curves and expressed as $\mathrm{pg} \mathrm{ml}^{-1}$.

\section{Cell viability, redox status and phase 2 enzyme activity}

Lactate dehydrogenase (LDH) in spent media was measured [26] to determine the effects of the different treatments on eukaryotic cell viability. Release of total thiols [GSHtot, GSH + glutathione disulfide (GSSG)], GSH and GSSG concentrations in cytosolic extracts were quantified using the 5,5'-dithionitrobenzoic acid (DTNB)-GSSG reductase recycling method [27]. Upon normalization to 
protein content, intracellular GSH and GSSG were expressed as nmoles $\mathrm{mg}^{-1} \mathrm{~min}^{-1}$. The extracellular thiol level was expressed as nmoles $\min ^{-1}$. NQO1 and GST activities were measured in cytosolic extracts as previously described [28], and the obtained values were normalized to the protein content and expressed as nmoles 1-chloro2,4-dinitrobenzene (CDNB) $\mathrm{mg}^{-1} \mathrm{~min}^{-1}$ and nmoles NAD $\mathrm{mg}^{-1} \mathrm{~min}^{-1}$, respectively.

\section{Statistical analysis}

Statistical significance was determined by $t$-test or ANOVA using the GraphPad PRISM 4.0 software (GraphPad Software, Inc., La Jolla, CA). A P-value of 0.05 or less was considered to be significant.

\section{Results}

Probiotic properties of L. gasseri OLL2809 and L13-la

L. gasseri OLL2809 and L13-Ia have been isolated from human intestine and raw bovine milk, respectively, and their properties have previously been reported [22,23]. To further assess these strains' probiotic features, we focused on their antimicrobial activity. Table 1 shows the inhibition halos produced by L13-Ia and OLL2809 against four pathogenic bacterial strains. The supernatants of both strains were found to be effective against all tested pathogens without significant differences in their inhibitory activity. This indicated that the two strains of L. gasseri exhibited similar antimicrobial behavior that, in absence of deeper investigation, could derive by lactic acid production or hydrogen dioxide, both molecules usually released by lactobacilli during their growth. Table 2 summarizes salient characteristics of OLL2809 and L13-Ia.

\section{Differential effects of $L$. gasseri strains on mature DCs}

Intestinal DCs are able to directly sample luminal antigens by extruding dendrites between epithelial cells $[3,29]$. To reproduce this interaction in vitro, we pulsed bone marrow-derived DCs ( $\geq 80 \% \mathrm{CD} 11 \mathrm{c}^{+}$) with LPS to obtain mature DCs (mDCs). Maturation was characterized by an increase in $C D 11 b^{+} C D 11 c^{+}$DCs (Figure 1A-B). These cells were cultured for $24 \mathrm{~h}$ in the presence of irradiated L. gasseri. L13-Ia, but not OLL2809, decreased the number of CD11b CD11c double-positive mDCs (32 and $52 \%$, respectively, Figure $1 \mathrm{C}-\mathrm{D})$. LPS treatment also caused an increase in the expression of the CD80 and CD40 costimulatory markers (Figure 1E-F). OLL2809, but not L13-Ia, increased the expression of both CD80 and CD40 on mDCs (Figure 1G-H). We next analyzed the effects of irradiated bacteria on the cytokine profile of the DCs. As previously reported [18], LPS induced maturation of DCs derived from this mouse strain and increased the secretion of IL-12 and TNF- $\alpha$, but not of IL-10 (Figure 2). Notably, in vitro challenge with both bacterial strains dramatically enhanced the expression of all examined cytokines including IL-10, showing significant differences with the positive control (mDCs alone; Figure 2).

\section{Stimulatory activity of $L$. gasseri strains on IECs}

Next, the capacity of OLL2809 and L13-Ia to stimulate enterocytes was investigated. Confluent monolayers of the murine epithelial cell line MODE-K were challenged with irradiated bacteria. IEC viability, evaluated by measuring LDH release in the medium, was not influenced by incubation with bacteria (data not shown). MODE-K cells were then analyzed to determine surface expression of MHC II molecules and secretion of the cytokine IL-6. FACS analysis showed that only L13-Ia induced MHC II expression (Figure 3A). However, both strains induced IL-6 secretion, although the levels of secretion were significantly different (Figure 3B). Interestingly, IL-6 production was also induced by metabolites secreted by OLL2809 but not by L13-Ia (Figure 3B).

The analysis of oxidative stress markers indicated a significant decline in intracellular GSH (Figure 3C upper panel) and the lack of a detectable alteration in GSSG content (data not shown) in cells incubated with both strains of L. gasseri. However, a significant increase in GSHtot release resulted from MODE-K cell treatment with the L13-Ia strain compared to the control culture (Figure 3C lower panel).

\section{Modulation of IEC-iDC interaction}

To evaluate the ability of IECs challenged by L. gasseri to instruct DCs, iDCs were incubated for $24 \mathrm{~h}$ with

Table 1 Antimicrobial activity of Lactobacillus gasseri L13-la and OLL2809 as determined by diffusion techniques

\begin{tabular}{|c|c|c|c|c|c|c|c|c|c|}
\hline \multirow{3}{*}{ Microorganisms } & \multicolumn{9}{|c|}{ Inhibition halo $(\mathrm{mm} \pm \mathrm{SD})$} \\
\hline & \multicolumn{3}{|c|}{ L13-la culture supernatant ( $\mu \mathrm{l} /$ disc) } & \multicolumn{3}{|c|}{ OLL2809 culture supernatant ( $\mu \mathrm{l} /$ disc) } & \multirow{2}{*}{$\begin{array}{c}\text { DMSO } \\
\text { ( } \mu \mathrm{l} / \mathrm{disc}) \\
20\end{array}$} & \multirow{2}{*}{$\begin{array}{c}\text { Gentamycin } \\
\text { ( } \mu \mathrm{g} / \mathrm{disc}) \\
\mathbf{8}\end{array}$} & \multirow{2}{*}{$\begin{array}{c}\text { Tetracycline } \\
\text { ( } \mu \mathrm{g} / \text { disc }) \\
7\end{array}$} \\
\hline & 5 & 10 & 20 & 5 & 10 & 20 & & & \\
\hline B. cereus DSM 4313 & $4.5 \pm 0.5$ & $6.5 \pm 0.5$ & $8 \pm 0.5$ & $4.5 \pm 0.5$ & $6.5 \pm 0.15$ & $8 \pm 0.35$ & na & $15.3 \pm 0.65$ & $9.7 \pm 0.7$ \\
\hline B. cereus DMS 4384 & $5 \pm 0.0$ & $6.5 \pm 0.0$ & $7.5 \pm 0.0$ & $4.5 \pm 0.15$ & $6.5 \pm 0.0$ & $8 \pm 0.15$ & na & $15.5 \pm 0.0$ & $9.65 \pm 0.15$ \\
\hline E. coli DMS 8579 & na & $3.45 \pm 0.45$ & $4.65 \pm 0.45$ & na & $3.5 \pm 0.4$ & $4.6 \pm 0.4$ & na & $15.7 \pm 0.4$ & $12.7 \pm 0.2$ \\
\hline Ps. aeruginosa & na & $4.65 \pm 0.15$ & $7.5 \pm 0.4$ & na & $4.65 \pm 0.2$ & $7.3 \pm 0.2$ & na & $5.7 \pm 0.2$ & $4.3 \pm 0.15$ \\
\hline
\end{tabular}

na, no activity. 
Table 2 Key characteristics of L.gasseri strains used in the study

\begin{tabular}{lccc}
\hline Strain & Code & Collection & Probiotic features \\
\hline OLL2809 & $\begin{array}{c}\text { 16S rRNA partial gene sequence available in } \\
\text { GenBank (accession number AB829518). }\end{array}$ & $\begin{array}{c}\text { Meiji Co, Ltd, } \\
\text { (Odawara, Japan) }\end{array}$ & $\begin{array}{c}\text { Colonization of human gut; activity in reducing lgE-mediated } \\
\text { allergy; growth inhibition of pathogenic species. }\end{array}$ \\
$\begin{array}{ccc}\text { L13-la } \\
\text { 16S rRNA partial gene sequence available in in issue } \\
\text { GenBank (accession number KF934204). }\end{array}$ & $\begin{array}{c}\text { ISPA-CNR (Italy) } \\
\text { Survival to gastric and pancreatic juice treatments; resistance } \\
\text { to bile salts; growth inhibition of pathogenic species. }\end{array}$ & \begin{tabular}{c} 
[23], this issue \\
\hline
\end{tabular}
\end{tabular}

media conditioned by MODE- $\mathrm{K}$ monolayers in the presence or absence of L. gasseri strains. Following this treatment, iDCs were LPS pulsed and cultured for additional $24 \mathrm{~h}$. As reported above, LPS increased expression of both CD80 and CD40 surface markers on DCs (Figure 4A-B). Pretreatment of DCs with supernatant from MODE-K monolayers (SupMODE) down-regulated the expression of these markers (Figure 4C). However, down-regulation was completely reversed when MODE-K cells were stimulated with TNF- $\alpha$ (Figure 4D). Interestingly, bacteriaconditioned supernatants from MODE-K cells induced a further increase in the expression of the co-stimulatory markers (Figure 4E-F). The data reported in Figure 4G and $\mathrm{H}$ clearly showed that inductive effects also resulted from metabolites secreted into the medium by both bac-

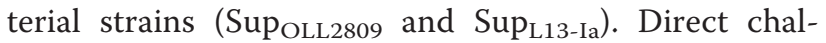
lenge with bacteria was much less effective than challenge with the bacterial metabolites in inducing the expression of CD80 and CD40 on DCs following LPS stimulation (Figure 4I-J). We next examined the effects of conditioned media on the cytokine profile. Interestingly,

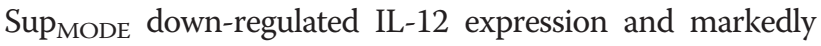
induced TNF- $\alpha$ and IL-10 in LPS-pulsed iDCs (Figure 5); this effect was dramatically reduced when MODE-K cells were treated with TNF- $\alpha$. Notably, media from bacteriaconditioned MODE-K cell cultures completely suppressed the expression of all examined cytokines. A similar effect was reproduced when DCs were treated with SupolL2809 and $\operatorname{Sup}_{\mathrm{L} 13-\mathrm{Ia}}$ (Figure 5). Baseline levels of IL-12, IL-10 and TNF- $\alpha$ in the various supernatants were undetectable, with the exception of TNF- $\alpha->$ Sup $_{\text {MODE }}$ where TNF- $\alpha$ levels were not significantly different from those found in the control (iDCs alone; data not shown). This indicated that added TNF- $\alpha\left(5 \mu \mathrm{g} \mathrm{l}^{-1}\right)$ was mainly metabolized/degraded after $24 \mathrm{~h}$ in this sample. Direct incubation of iDCs with irradiated bacteria dramatically enhanced the secretion of all examined cytokines, after LPS pulse, at levels comparable to those reported in Figure 2 (data not shown).

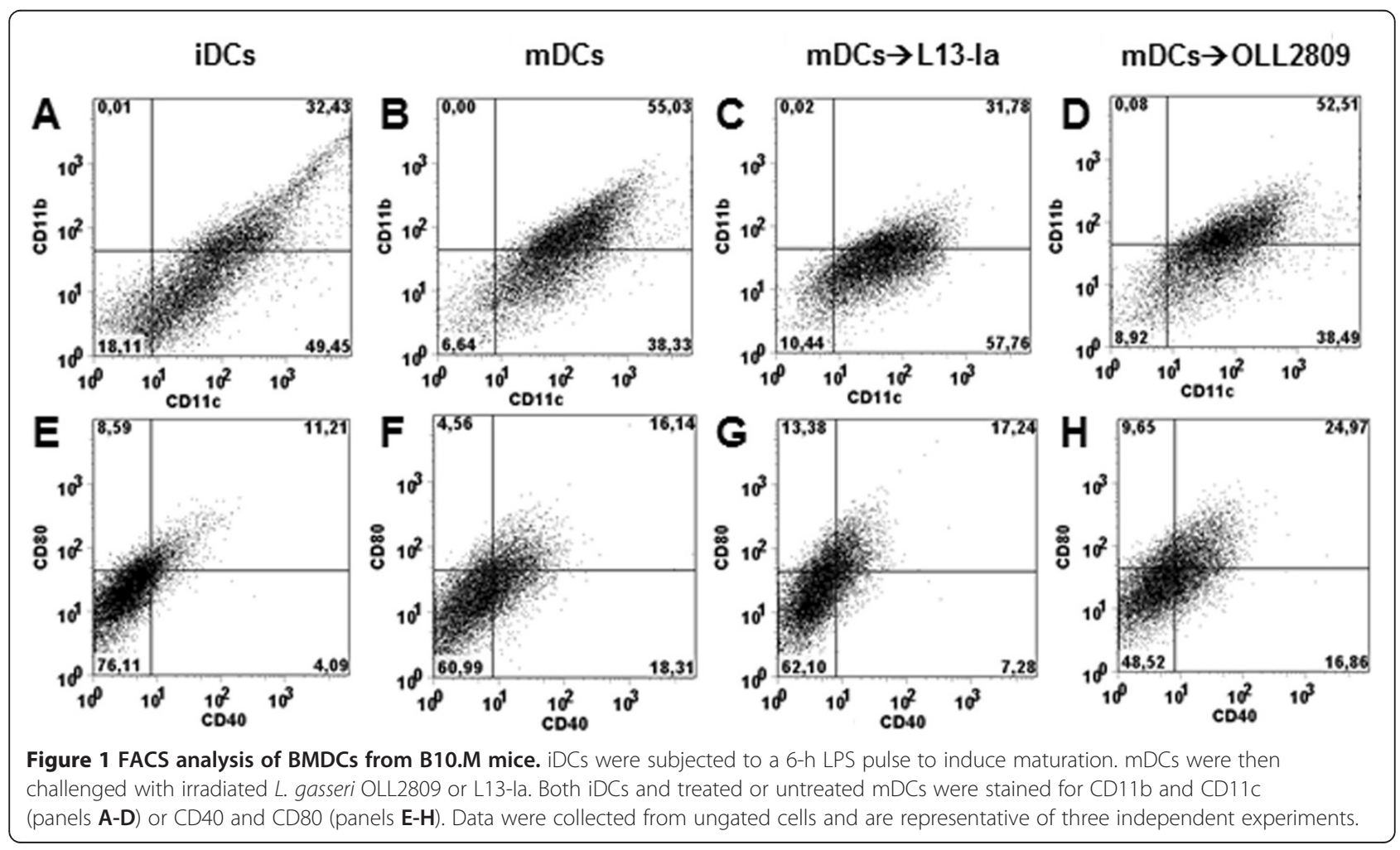



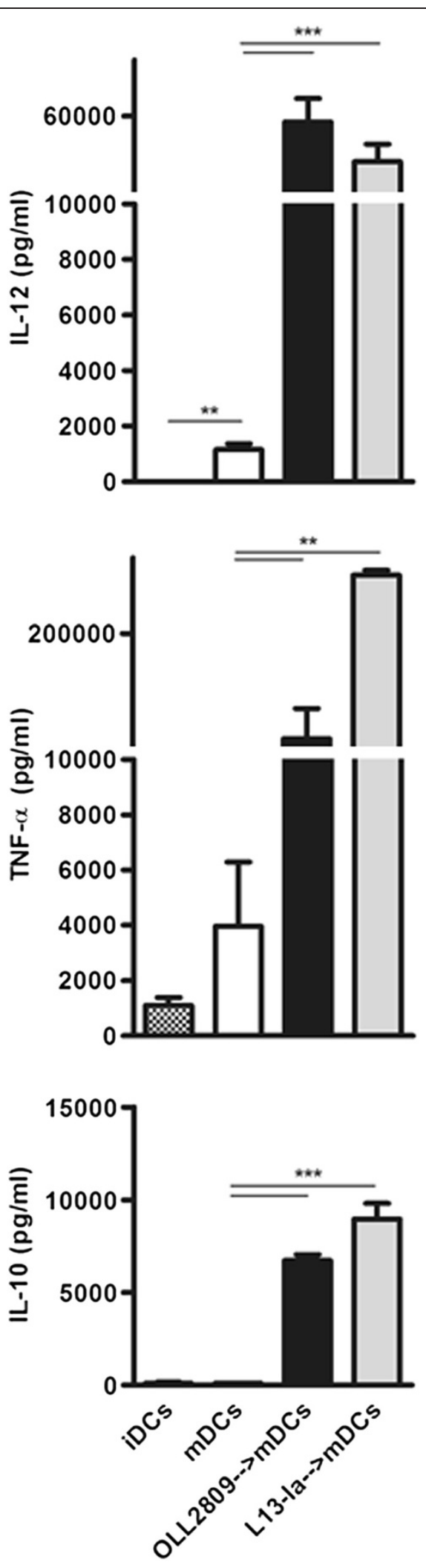

Figure 2 Cytokine production by $\mathrm{mDCs}$ in response to irradiated L. gasseri OLL2809 or L13-la. Culture supernatants were collected after $24 \mathrm{~h}$ and analyzed for IL-12, TNF- $a$ and IL-10 expression by sandwich-type ELISA; values are expressed in pg/ml; columns represent the mean \pm SD and are representative of three independent experiments. ${ }^{* *}, P<0.01$; ${ }^{* *}, P<0.001$.

\section{L. gasseri strains influence the antioxidant/cytoprotective defenses of DCs}

The effects on DC redox status and Nrf2-mediated cytoprotection elicited by the two L. gasseri strains were evaluated using LPS-pulsed DCs. In contrast to what was observed in IECs, a significant increase in intracellular GSH resulted from DC pre-exposure to OLL2809 compared to DCs treated with L13-Ia (Figure 6A), and GSH release in culture media was significantly increased by the presence of both L. gasseri strains (Figure 6A upper insert). Interestingly, significantly higher GST and NQO1 activities were found in DCs pre-exposed to both strains, although at different levels (OLL2809 > L13-Ia) (Figure 6B-C). When we examined the modulatory activities of bacteria-conditioned MODE-K cell culture on redox status and cytoprotective defenses, similar results were obtained, with the exception of a comparable increase of phase 2 enzyme activity operated by the two strains (Figure 6D-F). Importantly, SupMODE did not affect any of the examined antioxidant or cytoprotective parameters (Figure 6A-F). Finally, we examined the modulatory activities of SupolL2809 and Sup on antioxidant/cytoprotective defenses in DCs. Interestingly, intracellular GSH content, GSH release in culture media and phase 2 enzyme activity in DCs were significantly upregulated by stimulation with Supol22809 compared to stimulation with Sup $_{\text {L13-Ia }}$ (Figure 6G-I). These treatments had no detectable influence on DC viability or intracellular GSSG concentration (data not shown).

\section{Discussion}

In this study, we compared two probiotic strains of L. gasseri, OLL2809 and L13-Ia, and found that these strains had distinctive abilities to modulate in vitro mechanisms of innate immunity and antioxidant/detoxifying defenses.

OLL2809 was isolated from human feces [22]. The beneficial activity of this strain on mucosal inflammation has been previously shown in mice, where administration of OLL2809 was effective in reducing endometriotic lesions [30]. L13-Ia was isolated from raw whole bovine milk and was considered a potential probiotic strain [23] as it survived a selective in vitro digestion protocol. Another probiotic property of these strains has been confirmed in this study (Table 1).

The intestinal microbiota interacts with the local immune system promoting mechanisms of intestinal homeostasis [31]. Harnessing the contribution of probiotics to this physiological function has been proposed as a potential 


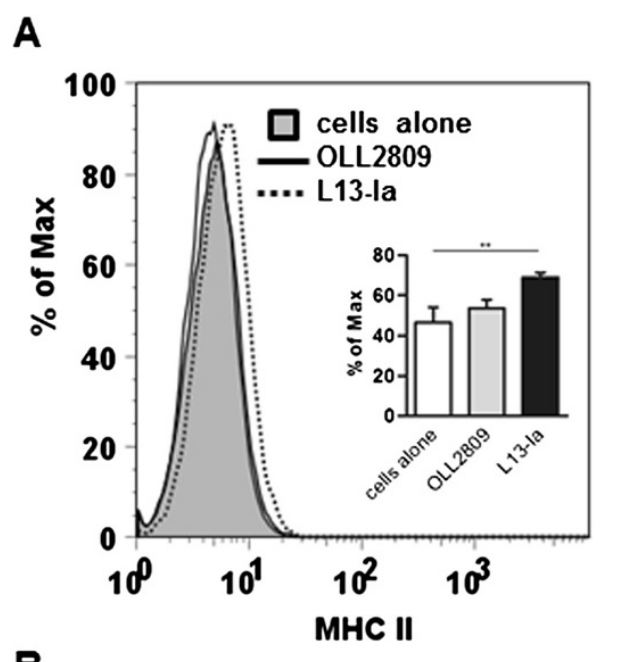

B

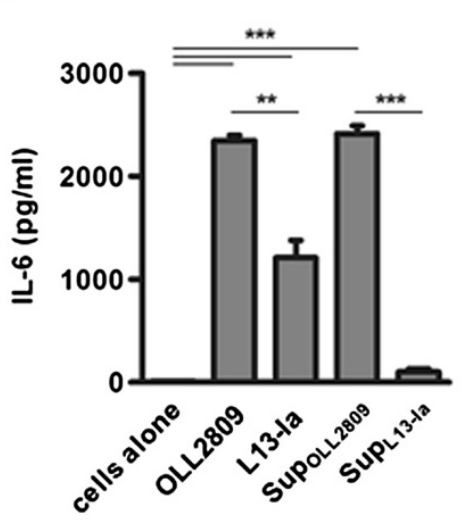

C
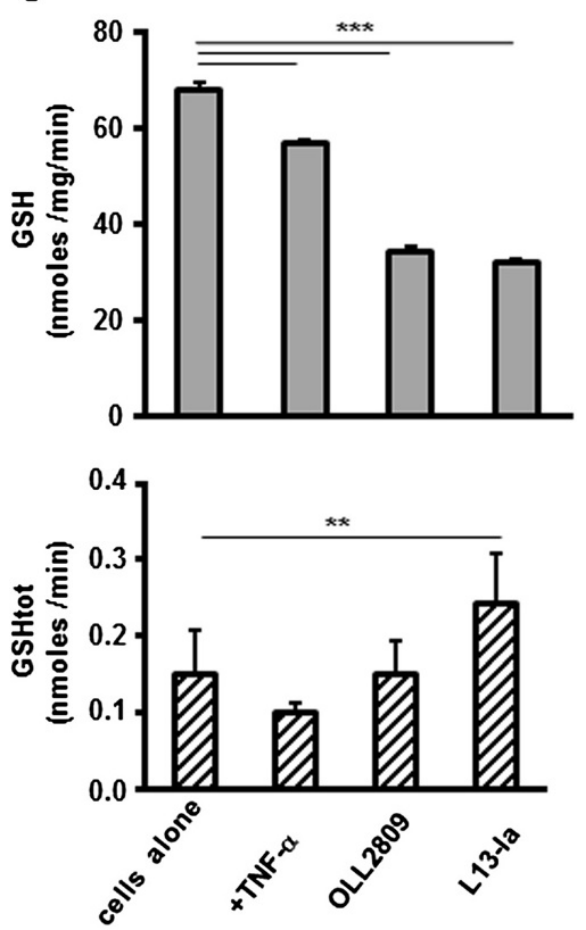

Figure 3 Effects of L. gasseri OLL2809 or L13-la on an intestinal cell line. A) FACS analysis of MHC class II expression in MODE-K cells incubated with irradiated L. gasseri OLL2809 or L13-la; values are expressed as percentages of the maximal fluorescence intensity. Inset, statistical evaluation of MHC class II expression; columns represent the mean \pm SD of three independent experiments; ${ }^{* *}, P<0.01$. B) IL-6 production by MODE-K cells following $24 \mathrm{~h}$ stimulation with irradiated bacteria or their metabolites (SupOLL2809 and SupL13-la); values are expressed in pg/ml. C) Intracellular GSH concentration in MODE-K cells, expressed in nmoles/mg prot/min (upper panel), and GSHtot amount in spent media, expressed in nmoles/min (lower panel), following $24 \mathrm{~h}$ stimulation with irradiated bacteria; columns represent the mean \pm SD and are representative of three independent experiments. sup, supernatant from irradiated bacteria incubated for $24 \mathrm{~h}$ in $\mathrm{RPMl}$ complete medium. ${ }^{* *}, P<0.01 ;{ }^{* * *}, P<0.001$.

beneficial treatment for inflammatory bowel disease [32]. The activity of these probiotic organisms is thought to be mediated by the interaction of microbeassociated molecular patterns (MAMPs) with pattern recognition receptors (PRRs) on antigen-presenting cells. In particular, the immune response against lactobacilli is dictated by conserved MAMPs [33]. As a result of these interactions, some L. gasseri strains induce DCs to produce high levels of IL-10, IL-6, IL-12, and TNF- $\alpha$ [33]. In line with these data, herein we showed that direct exposure of L. gasseri strains to DCs resulted in strong cytokine responses with no deviation toward a specific phenotype. Notably, the reported proinflammatory phenotype of $\mathrm{mDCs}$ derived from this mouse strain [34] was abrogated after challenge with both L. gasseri strains as IL-10 was also induced. Nevertheless, all of these cytokines may contribute to innate immunity by inducing the proliferation and differentiation of natural killer cells in vivo [35].

In functional experiments, we set the bacteria: eukaryotic cell ratio to $30: 1$ on the basis of a study showing that this proportion was optimal to stimulate cells [36]. Using this protocol, a differential activity of the two L. gasseri strains was shown following bacteria challenge of mature DCs. This in vitro condition resembles the physiologic interaction occurring between bacteria and DC protrusions across the intestinal epithelium that reflects an active response to local commensal flora and bacterial products [29]. In our experiments, the percentage of

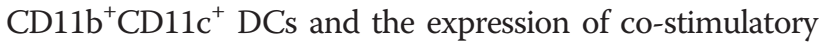
markers (CD40 and CD80) were increased following maturation. Intestinal lamina propria (LP) DCs are classified into $C D 11 c^{\text {hi }} C D 11 b^{\text {hi }}$ and CD $11 c^{\text {hi }} C D 11 b^{\text {lo }}$ DCs [37], which were found to be equivalent to $\mathrm{CD}_{103}{ }^{+} \mathrm{CD} 8 \alpha^{-}$and 


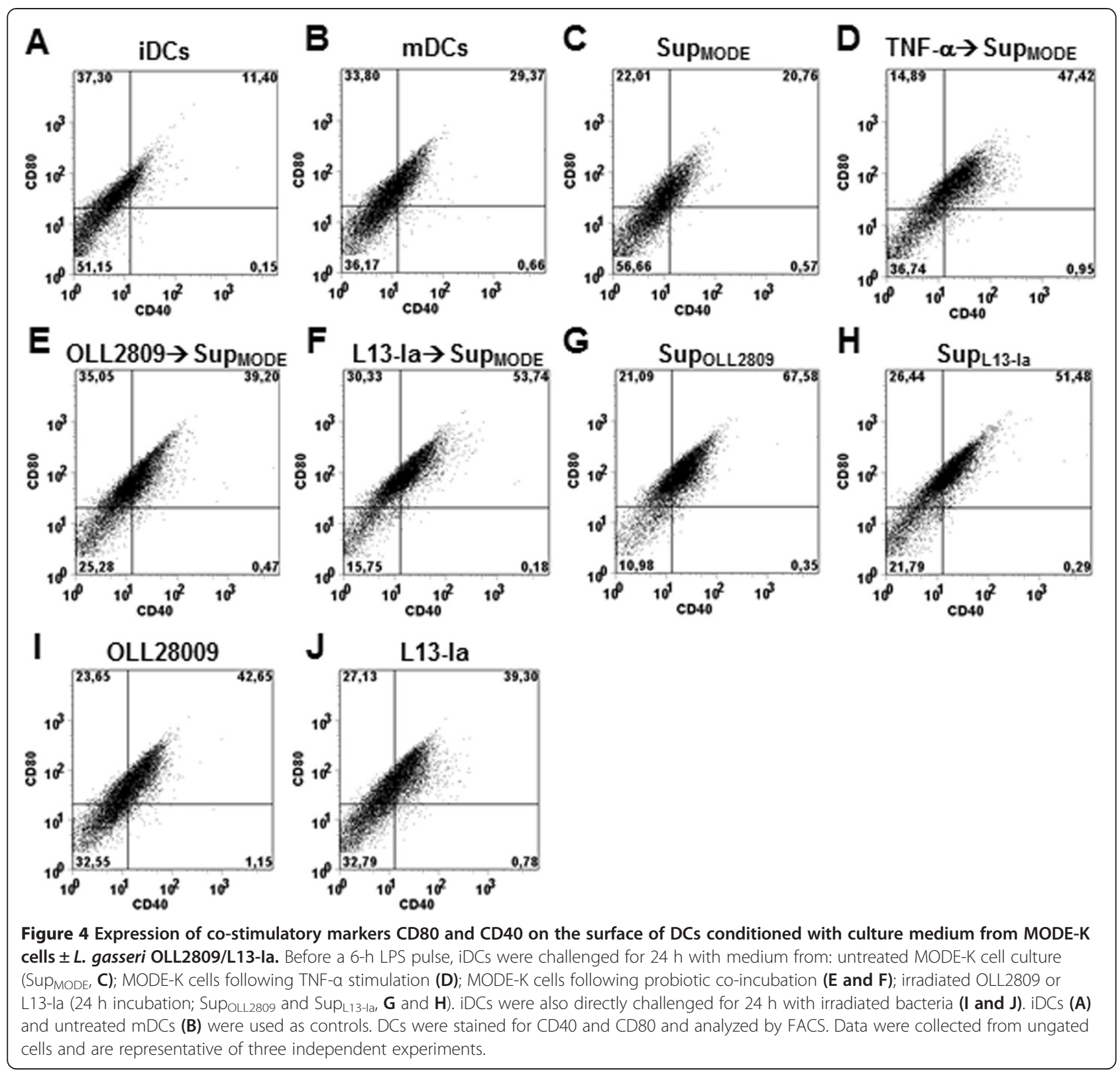

$\mathrm{CD} 103^{+} \mathrm{CD} 8 \alpha^{+}$LPDCs subsets, respectively [38]. Interestingly, only OLL2809 sustained maturation of DCs in our experiments, leaving unchanged the percentage of

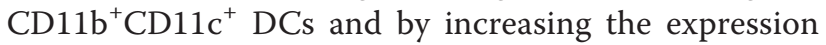
of co-stimulatory markers.

We also examined the interaction of L. gasseri strains with intestinal epithelial cells (IECs). IECs, in addition to their metabolic functions, play a major role in the generation of innate immunity. To explore this function of IECs, we used a murine epithelial cell line (MODE-K) derived from the small intestine [24]. We found that the two L. gasseri strains differentially influenced MODE-K cells. In particular, OLL2809 was more effective than L13-Ia in stimulating IL-6 secretion without inducing surface expression of MHC class II molecules. However, L13-Ia induced the expression of MHC class II, a phenomenon that allows IECs to stimulate $\mathrm{CD}^{+} \mathrm{T}$ cells during inflammation or in response to infection. Moreover, only SupolL2809 induced IL-6 secretion in MODE-K cells, thus further highlighting the existence of distinctive responses elicited by these strains. The biological significance of the IL-6 increase remains controversial because this cytokine has both pro-and anti-inflammatory activities. Its receptor, IL-6R, is expressed on the surface of only a few cell types including hepatocytes and some leukocytes. The IL-6/IL-6R complex associates with gp130, which dimerizes and initiates intracellular signaling that triggers anti-inflammatory activities, such as inhibition of apoptosis and a parallel 


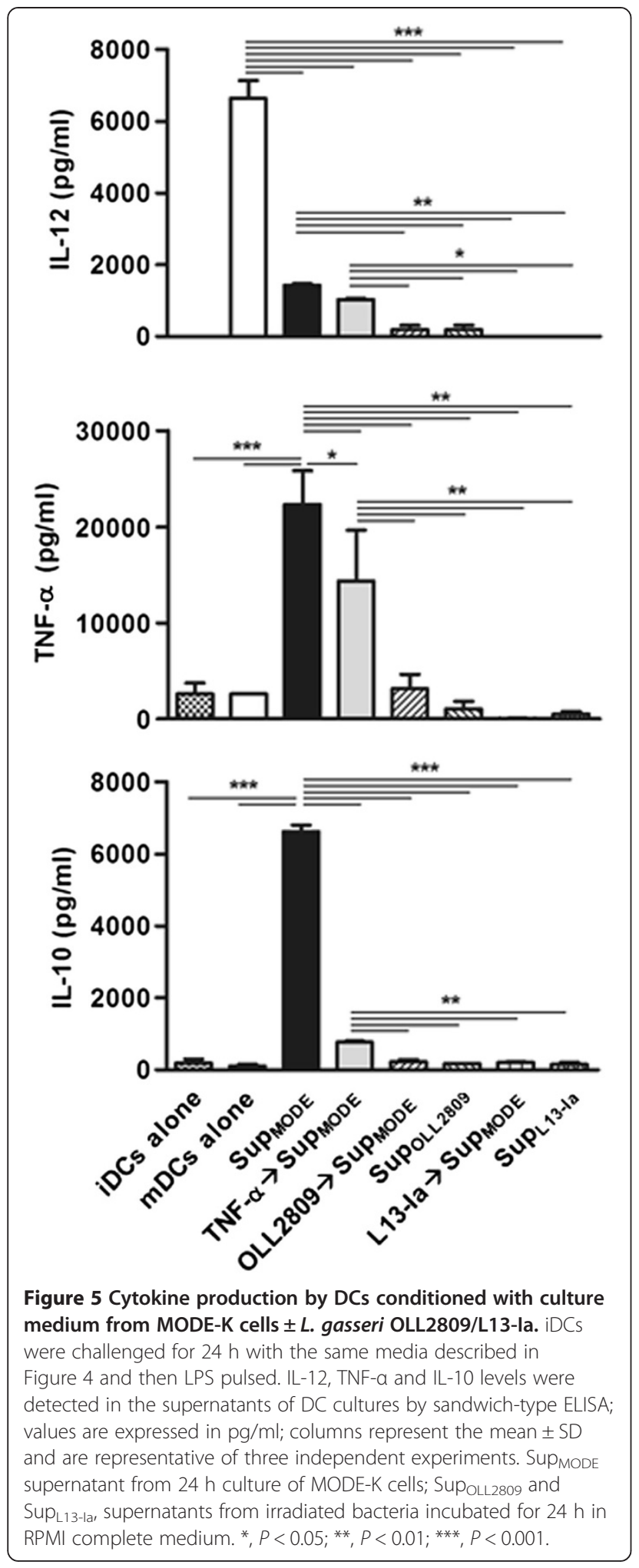

induction of proliferation in IECs [39]. However, IL-6 trans-signaling appears to mediate the pro-inflammatory activity of this cytokine, a process involving the binding of the soluble form of IL-6R to gp130 on cells that do not express IL-6R [39]. Our findings suggest that OLL2809 might contribute to gut immune homeostasis better than L13-Ia. Moreover, our results strengthen the concept that a probiotic activity can be induced not only from whole microorganisms and cell wall components but also from secreted metabolites. Stabilization of the enterocyte cytoskeleton was found to be mediated by a protease-sensitive metabolite secreted by the probiotic mixture VSL\#3 [40]. More recently, exposure to probiotic-conditioned media was shown to attenuate the inflammatory responses induced in different enterocyte models [41].

In the intestinal lamina propria, DCs are classically immature DCs that, following antigen encounter, migrate into mesenteric lymph nodes where they are primed. The existence of IEC-DC crosstalk has been suggested by observations showing that IECs can drive differentiation of Treg cell-promoting DCs. This differentiation is mediated by IEC-secreted transforming growth factor- $\beta$ and retinoic acid [42]. In agreement with these findings, we confirmed that medium conditioned by unstimulated MODE-K cells induced a regulatory phenotype in DCs, as shown by the reduced surface expression of co-stimulatory markers and, most importantly, reversal of the IL-12/IL-10 ratio. In the presence of a pro-inflammatory stimulus (i.e., treatment with TNF- $\alpha$ ), this regulatory phenotype was abrogated, confirming that IEC-DC crosstalk is highly regulated. We further addressed this issue by evaluating the ability of $L$. gasseri strains to modulate the IEC-DC interaction. Our data indicate that both strains influenced IEC-DC crosstalk with distinct outcomes compared to those induced by Sup MODE. In particular, these strains markedly enhanced the expression of co-stimulatory markers and downregulated IL-12, TNF- $\alpha$ and IL-10 secretions by mDCs. In addition, similar alterations were induced by SupolL2809

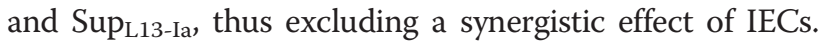
However, our model does not allow us to further elucidate this probiotic activity because MODE-K cells do not form a confluent monolayer, instrumental to analyze the different roles played by paracellular and transcytosis pathways [42]. Taken together, our data suggest that the L. gasseri influence on IEC-DC crosstalk is dominant over IEC activity. Importantly, MODE-K cells and L. gasseri are able to produce different outcomes, regulatory $\mathrm{mDCs}$ and "lowresponsive" $\mathrm{mDCs}$, respectively.

Another beneficial effect on host immunity arising from the interaction between epithelia and commensal bacteria is the generation of reactive oxygen species that may activate the Nrf2 pathway and lead to improved antioxidant/ detoxifying defenses [6]. The Nrf2-Keap1 complex serves as an intracellular oxidative stress sensor, and Nrf2 release, triggered by mild ROS production, activates the synthesis of a battery of cytoprotective/defensive proteins including GSH, GST and NQO1 that protect cells against oxidative stress and promote cell survival [5]. GSH plays a key role 


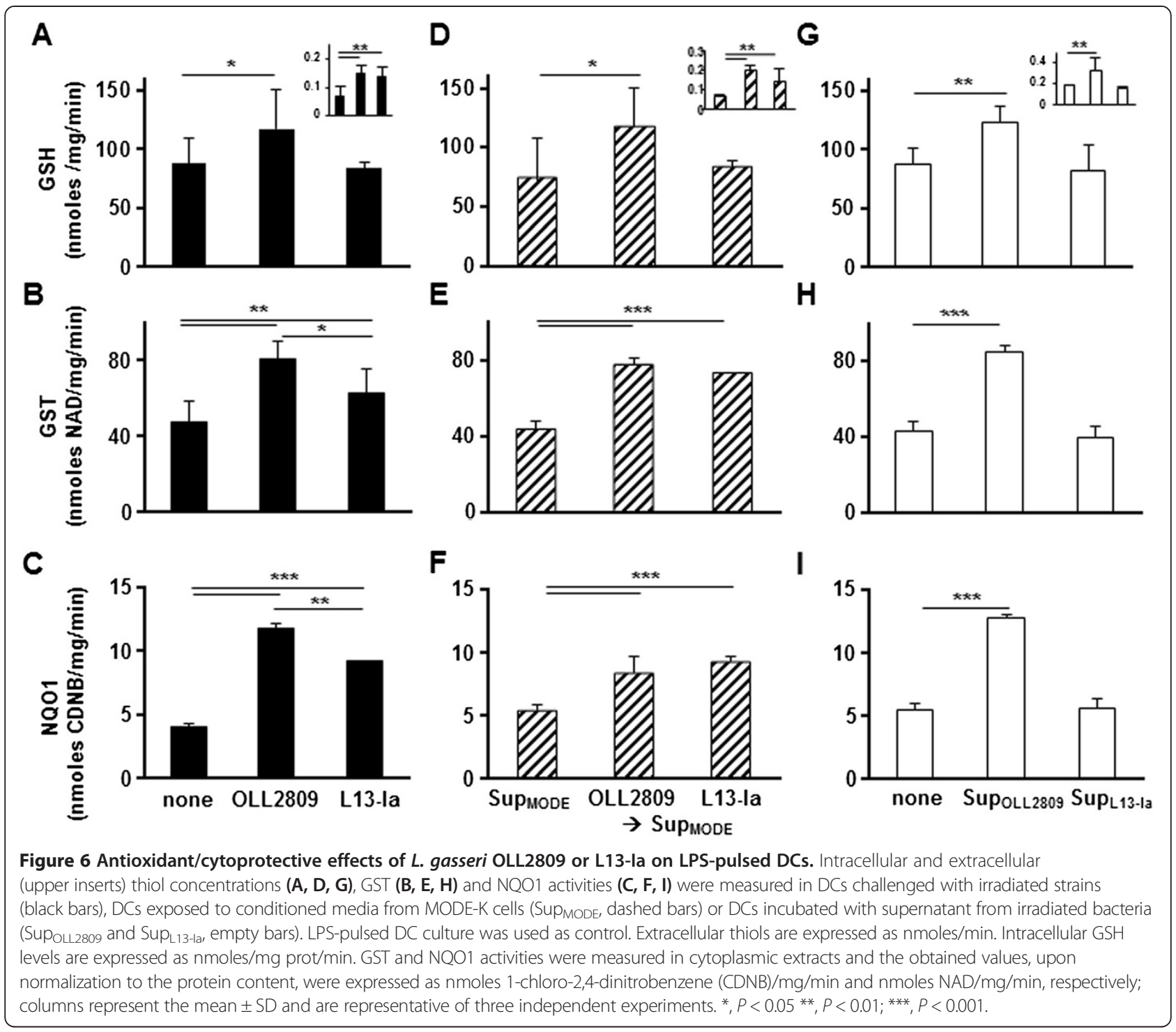

in the maintenance and regulation of the cell's redox status. Our data showing opposing effects of bacterial strains on MODE-K cells' and DCs' intracellular GSH content are consistent with the reported pro-oxidant activity exhibited by probiotic strains [6] and with the improved ability of DCs to survive in an oxidant-rich environment [43]. Under normal conditions, intracellular GSH levels depend upon the rates of GSH synthesis/ oxidation and on GSH export from cells, and the GSH/ GSSG pair is widely used as an indicator of redox status. Data from this study on MODE-K cells, according to the literature, indicates that the lack of intracellular GSSG accumulation is associated with depletion and increased export of intracellular GSH [44]. In contrast, the increased intracellular GSH concentration accompanied by the increase in GSHtot export from the DCs without any measurable raise of intracellular GSSG concentration indicates the ability of DCs to respond to L. gasseri-induced oxidative stress by increasing GSH synthesis. These results, along with the results showing the improvement of GST and NQO1 activities in DCs directly exposed to L. gasseri strains or to conditioned supernatants from MODE-K cells, along with in vivo studies, further support the ability of bacterial strains to activate the Nrf-2 pathway $[8,9]$.

\section{Conclusions}

We have demonstrated in vitro differential immunomodulatory activities of two probiotic strains of L. gasseri, isolated from different sources. Our data also highlight the potential of secreted metabolites derived from these strains to influence IEC-DC crosstalk, Nrf2mediated cytoprotection and consequently, gut immune homeostasis. Whether bacterial cell wall breakdown 
products or secreted molecules were responsible for this phenomenon is under investigation to aid in applications aimed at the amelioration of specific immunological conditions.

\section{Abbreviations}

DCs: Bone marrow-derived dendritic cells; IECs: Intestinal epithelial cells; GSH: Glutathione; GSTs: Glutathione S-transferases; MAMPs: Microbialassociated molecular patterns; NQO1: NAD(P)H:quinone oxidoreductase; Nrf2: Nuclear erythroid 2-related factor 2; ROS: Reactive oxygen species; Sup mode: Supernatant from MODE-K monolayers; SupoLL2809/L13-1a: Supernatant from bacterial strains; $\vee G C L:$ Gamma-glutamylcysteine ligase.

\section{Competing interests}

The authors declare that they have no competing interests.

\section{Authors' contributions}

$D L, P B, S T$ and MR conceived the study; MR and ST designed the study; DL, $\mathrm{JM}, \mathrm{PB}$ and $\mathrm{FN}$ did the laboratory work; $\mathrm{DL}, \mathrm{JM}, \mathrm{PB}, \mathrm{FB}, \mathrm{TS}, \mathrm{ST}$ and $\mathrm{MR}$ analysed the data; $\mathrm{DL}, \mathrm{PB}$, and MR wrote the manuscript; all authors read and approved the final manuscript.

\section{Acknowledgments}

This work was supported by CNR grant under the Agreement of Scientific Cooperation CNR-JSPS 2010-11 and by CNR-CISIA 2011 grant.

\section{Author details}

${ }^{1}$ Institute of Food Sciences, NRC, Avellino, Italy. ${ }^{2}$ Department of Biofunctional Science and Technology, Graduate School of Biosphere Science, Hiroshima University, Higashi, Hiroshima, Japan. ${ }^{3}$ Institute of Sciences of Food Production, NRC, Bari, Italy. ${ }^{4}$ Division of Research and Development, Meiji Co., Ltd., Odawara, Japan.

Received: 14 October 2013 Accepted: 18 December 2013

Published: 23 December 2013

\section{References}

1. Borchers AT, Selmi C, Meyers FJ, Keen CL, Gershwin ME: Probiotics and immunity. J Gastroenterol 2009, 44:26-46.

2. Tlaskalova-Hogenova H, Stepankova R, Hudcovic T, Tuckova L, Cukrowska B, Lodinova-Zadnikova R, Kozakova H, Rossmann P, Bártová J, Sokol D, Funda DP, Borovská D, Reháková Z, Sinkora J, Hofman J, Drastich P, Kokesová A: Commensal bacteria (normal microflora), mucosal immunity and chronic inflammatory and autoimmune diseases. Immunol Lett 2004, 93:97-108.

3. Rescigno M, Urbano M, Valzasina B, Francolini M, Rotta G, Bonasio R, Granucci F, Kraehenbuhl JP, Ricciardi-Castagnoli P: Dendritic cells express tight junction proteins and penetrate gut epithelial monolayers to sample bacteria. Nat Immunol 2011, 2:361-367.

4. Kim J, Cha YN, Surh YJ: A protective role of nuclear factor-erythroid 2-related factor-2 (Nrf2) in inflammatory disorders. Mutat Res 2010, 690:12-23.

5. Niture SK, Khatri R, Jaiswal AK: Regulation of Nrf2-an update. Free Radic Biol Med 2013. doi:10.1016/j.freeradbiomed. 2013.02.008.

6. Kumar A, Wu H, Collier-Hyams LS, Hansen JM, Li T, Yamoah K, Pan ZQ, Jones DP, Neish AS: Commensal bacteria modulate cullin-dependent signaling via generation of reactive oxygen species. EMBO J 2007, 26:4457-4466.

7. Lin PW, Myers LE, Ray L, Song SC, Nasr TR, Berardinelli AJ, Kundu K, Murthy N, Hansen JM, Neish AS: Lactobacillus rhamnosus blocks inflammatory signaling in vivo via reactive oxygen species generation. Free Radic Biol Med 2009, 47:1205-1211.

8. Endo H, Niioka M, Kobayashi N, Tanaka M, Watanabe T: Butyrate-producing probiotics reduce nonalcoholic Fatty liver disease progression in rats: new insight into the probiotics for the gut-liver axis. PLoS One 2013, 8:e63388. doi:10.1371/journal.pone.0063388.

9. Gosai V, Ambalam P, Raman M, Kothari CR, Kothari RK, Vyas BR, Sheth NR: Protective effect of Lactobacillus rhamnosus 231 against N-Methyl-N'-nitro-N-nitrosoguanidine in animal model. Gut Microbes 2011, 2:319-325.

10. O'Hara AM, O'Regan P, Fanning A, O'Mahony C, Macsharry J, Lyons A, Bienenstock J, O'Mahony L, Shanahan F: Functional modulation of human intestinal epithelial cell responses by Bifidobacterium infantis and Lactobacillus salivarius. Immunology 2006, 118:202-215.
11. Haller D, Serrant P, Peruisseau G, Bode C, Hammes WP, Schiffrin E, Blum S: IL-10 producing CD14low monocytes inhibit lymphocyte-dependent activation of intestinal epithelial cells by commensal bacteria. Microbiol Immunol 2002, 46:195-205.

12. Rimoldi M, Chieppa M, Larghi P, Vulcano M, Allavena P, Rescigno M: Monocyte-derived dendritic cells activated by bacteria or by bacteriastimulated epithelial cells are functionally different. Blood 2005, 106:2818-2826

13. Banchereau J, Steinman RM: Dendritic cells and the control of immunity. Nature 1998, 392:245-252.

14. Huang Q, Liu D, Majewski P, Schulte LC, Korn JM, Young RA, Lander ES, Hacohen N: The plasticity of dendritic cell responses to pathogens and their components. Science 2001, 294:870-875.

15. Christensen HR, Frokiaer H, Pestka JJ: Lactobacilli differentially modulate expression of cytokines and maturation surface markers in murine dendritic cells. J Immunol 2002, 168:171-178.

16. Hart AL, Lammers K, Brigidi P, Vitali B, Rizzello F, Gionchetti P, Campieri M, Kamm MA, Knight SC, Stagg AJ: Modulation of human dendritic cell phenotype and function by probiotic bacteria. Gut 2004, 53:1602-1609.

17. Medina M, Izquierdo E, Ennahar S, Sanz Y: Differential immunomodulatory properties of Bifidobacterium longum strains: relevance to probiotic selection and clinical applications. Clin Exp Immunol 2007, 150:531-538.

18. Menard O, Batel MJ, Gaboriau-Routhiau V, Waligora-Dupriet AJ: Gnotobiotic mouse immune response induced by Bifidobacterium sp. strains straind from infants. Appl Environ Microbiol 2008, 74:660-666.

19. D'Arienzo R, Maurano F, Lavermicocca P, Ricca E, Rossi M: Modulation of the immune response by probiotic strains in a mouse model of gluten sensitivity. Cytokine 2009, 48:254-259.

20. D'Arienzo R, Bozzella G, Rossi M, De Bellis P, Lavermicocca P, Sisto A: Distinct immunomodulatory properties of Lactobacillus paracasei strains. J Appl Microbiol 2011, 111:1482-1491.

21. Selle K, Klaenhammer TR: Genomic and phenotypic evidence for probiotic influences of Lactobacillus gasseri on human health. FEMS Microbiol Rev 2013, 37:915-935.

22. Sashihara T, Sueki N, Ikegami $\mathrm{S}$ : An analysis of the effectiveness of heat-killed lactic acid bacteria in alleviating allergic diseases. J Dairy Sci 2006, 89:2846-2855.

23. Baruzzi F, Poltronieri P, Quero GM, Morea M, Morelli L: An in vitro protocol for direct isolation of potential probiotic lactobacilli from raw bovine milk and traditional fermented milks. Appl Microbiol Biotechnol 2011, 90:331-342.

24. Vidal K, Grosjean I, Revillard JP, Gespach C, Kaiserlian DJ: Immortalization of mouse intestinal epithelial cells by the SV40-large T gene. Phenotypic and immune characterization of the MODE-K cell line. Immunol Methods 1993, 166:63-73.

25. Lutz MB, Kukutsch N, Ogilvie AL, Rossner S, Koch F, Romani N, Schuler G: An advanced culture method for generating large quantities of highly pure dendritic cells from mouse bone marrow. J Immunol Methods 1999, 223:77-92.

26. Luongo D, Severino L, Bergamo P, De Luna R, Lucisano A, Rossi M: Interactive effects of fumonisin B1 and alpha-zearalenol on proliferation and cytokine expression in Jurkat T cells. Toxicol In Vitro 2006, 20:1403-1410.

27. Bergamo P, Gogliettino M, Palmieri G, Cocca E, Maurano F, Stefanile R, Balestrieri M, Mazzarella G, David C, Rossi M: Conjugated linoleic acid protects against gliadin-induced depletion of intestinal defenses. Mol Nutr Food Res 2011, 55:S248-S256.

28. Bergamo P, Maurano F, Rossi M: Phase 2 enzyme induction by conjugated linoleic acid improves lupus-associated oxidative stress. Free Radic Biol Med 2007, 43:71-79.

29. Chieppa M, Rescigno M, Huang AYC, Germain RN: Dynamic imaging of dendritic cell extension into the small bowel lumen in response to epithelial cell TLR engagement. J Exp Med 2006, 203:2841-2852.

30. Itoh H, Sashihara T, Hosono A, Kaminogawa S, Uchida M: Lactobacillus gasseri OLL2809 inhibits development of ectopic endometrial cell in peritoneal cavity via activation of NK cells in a murine endometriosis model. Cytotechnology 2011, 63:205-210.

31. Cerf-Bensussan N, Gaboriau-Routhiau V: The immune system and the gut microbiota: friends or foes? Nat Rev Immunol 2010, 10:735-744.

32. Gourbeyre P, Denery S, Bodinier M: Probiotics, prebiotics, and synbiotics: impact on the gut immune system and allergic reactions. J Leukoc Biol 2011, 89:685-695. 
33. Stoeker L, Nordone S, Gunderson S, Zhang L, Kajikawa A, LaVoy A, Miller M, Klaenhammer TR, Dean GA: Assessment of Lactobacillus gasseri as a candidate oral vaccine vector. Clin Vaccine Immunol 2011, 18:1834-1844.

34. Bergamo P, Maurano F, D'Arienzo R, David C, Rossi M: Association between activation of phase 2 enzymes and down-regulation of dendritic cell maturation by $\mathrm{C} 9$, $\mathrm{t} 11$-conjugated linoleic acid. Immunol Lett 2008 117:181-190.

35. Kawase M, He F, Kubota A, Yoda K, Miyazawa K, Hiramatsu M: Heat-killed Lactobacillus gasseri TMC0356 protects mice against influenza virus infection by stimulating gut and respiratory immune responses. FEMS Immunol Med Microbiol 2012, 64:280-288.

36. Ruiz PA, Hoffmann M, Szcesny S, Blaut M, Haller D: Innate mechanisms for Bifidobacterium lactis to activate transient pro-inflammatory host responses in intestinal epithelial cells after the colonization of germ-free rats. Immunology 2005, 115:441-450.

37. Uematsu S, Fujimoto K, Jang MH, Yang BG, Jung YJ, Nishiyama M, Sato S, Tsujimura T, Yamamoto M, Yokota Y, Kiyono H, Miyasaka M, Ishii K, Akira S: Regulation of humoral and cellular gut immunity by lamina propria dendritic cells expressing Toll-like receptor 5. Nat Immunol 2008, 9:769-776.

38. Fujimoto K, Karuppuchamy T, Takemura N, Shimohigoshi M, Machida T, Haseda Y, Aoshi T, Ishii K, Akira S, Uematsu S: A new subset of CD103+ CD8a + dendritic cells in the small Intestine expresses TLR3, TLR7, and TLR9 and induces Th1 response and CTL activity. J Immunol 2011, 186:6287-6295.

39. Rose-John S: IL-6 trans-signaling via the soluble IL-6 receptor: importance for the Pro-inflammatory activities of IL-6. Int J Biol Sci 2012, 8:1237-1247.

40. Otte J-M, Podolsky DK: Functional modulation of enterocytes by gram-positive and gram-negative microorganisms. Am J Physiol Gastrointest Liver Physiol 2004, 286:G613-G626.

41. Ganguli K, Meng D, Rautava S, Lu L, Walker WA, Nanthakumar N: Probiotics prevent necrotizing enterocolitis by modulating enterocyte genes that regulate innate immune-mediated inflammation. Am J Physiol Gastrointest Liver Physiol 2013, 304:G132-G141.

42. Nliev ID, Mileti E, Matteoli G, Chieppa M, Rescigno M: Intestinal epithelial cells promote colitis-protective regulatory T-cell differentiation through dendritic cell conditioning. Mucosal Immunol 2009, 2:340-350.

43. Rivollier A, Perrin-Cocon L, Luche S, Diemer H, Strub JM, Hanau D, van Dorsselaer A, Lotteau V, Rabourdin-Combe C, Rabilloud T, Servet-Delprat C: High expression of antioxidant proteins in dendritic cells: possible implications in atherosclerosis. Mol Cell Proteomics 2006, 5:726-736.

44. Ballatori N, Krance SM, Notenboom S, Shi S, Tieu K, Hammond CL: Glutathione dysregulation and the etiology and progression of human diseases. Biol Chem 2009, 390:191-214.

doi:10.1186/1471-2180-13-298

Cite this article as: Luongo et al: Differential modulation of innate immunity in vitro by probiotic strains of Lactobacillus gasseri. BMC Microbiology 2013 13:298.

\section{Submit your next manuscript to BioMed Central and take full advantage of:}

- Convenient online submission

- Thorough peer review

- No space constraints or color figure charges

- Immediate publication on acceptance

- Inclusion in PubMed, CAS, Scopus and Google Scholar

- Research which is freely available for redistribution

Submit your manuscript at www.biomedcentral.com/submit 